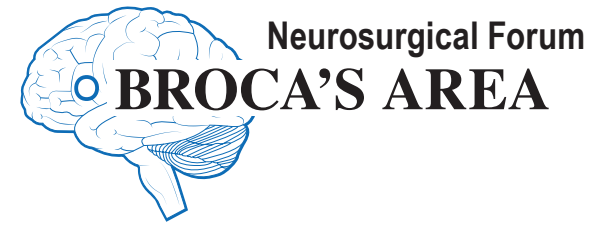

\section{How did Michael Jackson challenge our understanding of spine biomechanics?}

Nishant S. Yagnick, MCh, Manjul Tripathi, MCh, and Sandeep Mohindra, $\mathrm{MCh}$

Department of Neurosurgery, Postgraduate Institute of Medical Education and Research, Chandigarh, India

I N 1987, a new music video, "Smooth Criminal," showed Michael Jackson (MJ, as he is known to his fans) performing a mind-boggling dance move. He leaned $45^{\circ}$ forward while keeping his spine straight, using only his feet to prevent him from falling (Fig. 1A). Those who believed that special effects had been used in the video were shocked to see this man, also known as the "Moonwalker," perform the move in live concerts all over the world. For generations since, dancers have tried to emulate his movements and have developed the dance form he introduced into a new challenge to our understanding of spinal biomechanics. But did MJ really achieve the impossible?

When the human body stands erect, its center of gravity (COG) lies in front of the second sacral vertebra. Studies of biomechanics and kinesiology suggest that, as we bend forward with a straight torso, keeping our hip joints as the fulcrum, the erector spinae muscles act like cables to support the suspended spinal column during the forward shift of the COG, preventing the body from falling forward. However, when the fulcrum for forward bending is shifted to the ankle joints, the erector spinae lose their ability to maintain the COG, and strain is shifted to the Achilles tendon (Fig. 1B). ${ }^{1}$ This allows for a very limited degree of forward bending from the ankle joints, while keeping a stiff straight posture-unless you are Michael Jackson. Most trained dancers with strong core strength will reach a maximum of $25^{\circ}$ to $30^{\circ}$ of forward bending while performing this action. MJ pulled off a gravity-defying $45^{\circ}$ move that seems unearthly to any witness. Several MJ fans, including the authors, have tried to copy this move and failed, often injuring themselves in their endeavors.

As much as we would like to believe that MJ broke
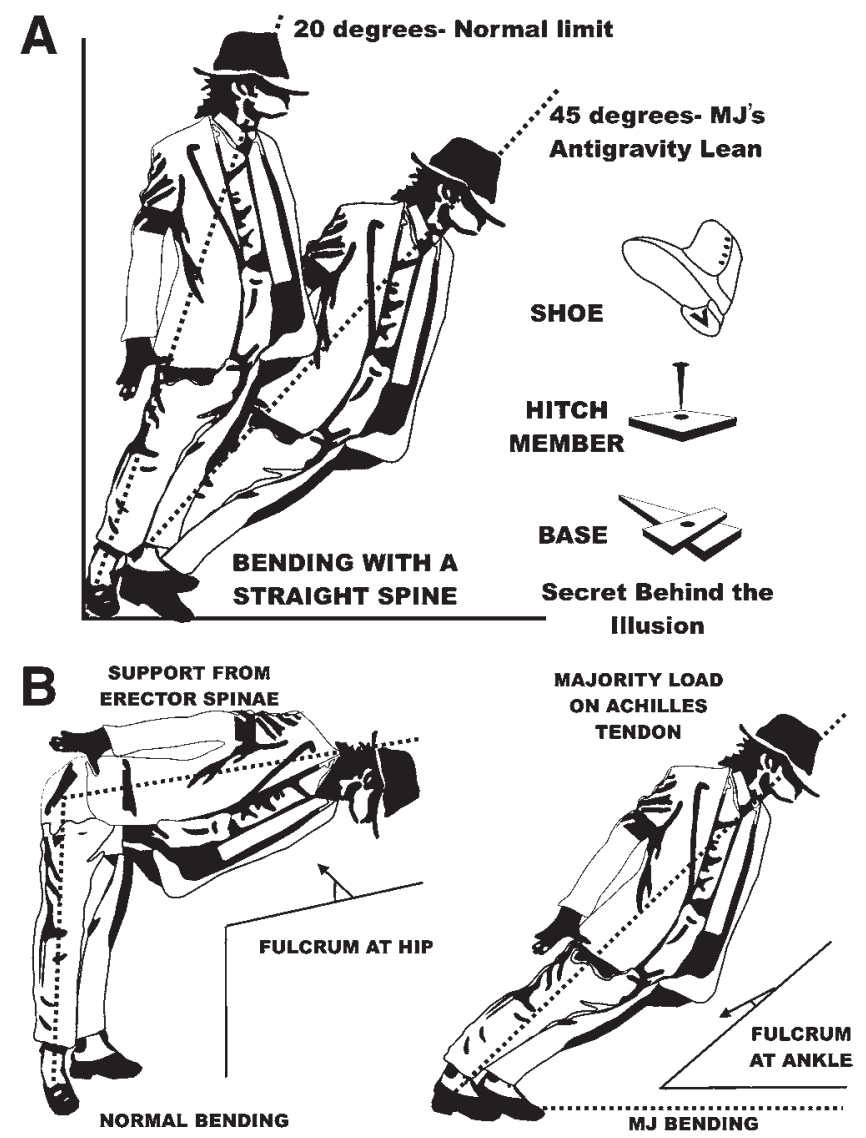

FIG. 1. A: Drawings showing the "antigravity tilt" ( $>45^{\circ}$ forward bend), the dance move introduced by Michael Jackson, in comparison to the normal limit of a human tilt ( $20^{\circ}$ forward bend), as well as the conceptualized shoe designed by MJ and coinventors. B: Shift of the fulcrum from the sacrum to the Achilles tendon in MJ's antigravity tilt. Copyright Manjul Tripathi. Published with permission. 
the relationship between physiology and physics, a patent registered under his name shows that the move was accomplished with a clever invention. Along with fellow inventors, MJ developed a special shoe that had a slot in the heel. The triangular slot could engage a hitch member (a metallic peg, which emerged from the stage floor at just the right time), allowing the dancer to obtain the right amount of extra support to be able to lean forward beyond physiological limits (Fig. 1A). ${ }^{2}$ Alas, MJ's move was an illusionary trick. But even with specially designed footwear and the support of the hitch member, the move is incredibly hard to pull off, requiring athletic core strength from strengthened spinal muscles and lower-limb antigravity muscles.

Trick or not, new forms of dancing inspired by MJ have begun to challenge our understanding of the modes and mechanisms of spinal injury. Ever since MJ entertained us with his fabulous moves, throughout the world dancers have tried to jump higher, stretch farther, and turn faster than ever before. The hip-hop style of dancing and, particularly its offshoot, b-boying/b-girling are two styles that include significant elements of acrobatics. Stresses on the spinal column experienced by dancers of those styles are very high and frankly a bit odd. The rapid rise in popularity of dance as an art and exercise the world over is bound to produce new forms of injuries that may perplex the neurosurgeon. The situation will become more complicated when a dancer, who has received a surgical construct for fixation, wants to continue dancing. The art of movement is life itself for many dancers, and it remains to be seen how surgical implants will hold up to the stresses of dance. While many new questions need answering, what is certain is that all this is owed to the ever-fresh inventiveness and style of "The King of Pop."

\section{Addendum}

At the time of publication, we focused our attention on the spinal biomechanics of Michael Jackson's live performances, in which he performed the "antigravity lean" by using a combination of his patented "shoe mechanism" along with intense core-strength training. Through this medium, we also commented on the evolution of challenging modern dance forms, the importance of training in dance to avoid injury, and the need for better end points of care in dancers who have received spinal constructs for injury. It has since been brought to our attention by Mr. Kevin Pike, the special effects consultant for the music video of the song "Smooth Criminal," which was filmed in 1987, that the story of the inception of the antigravity lean would be a welcome addition to the article. ${ }^{3}$ The addition of this information beautifully completes the picture of the inventiveness and ingenuity of the artists involved in the creation of the antigravity lean.

Mr. Pike provides a first-hand account of how the move was performed during shooting of the music video. ${ }^{3}$ There, an earlier type of shoe mechanism-not the innovative patented shoe technique we describe in this article-was used in combination with "fly belts" to shoot the dance move. These fly belts were attached to a complex system of piano wires and cables, which in turn, were controlled by overhead pulleys and special effects assistants during the shoot. At the time of the shoot, when the move was conceived, the fly-belts and cable mechanism constituted the primary weightbearing tool that allowed the antigravity lean to be recorded on video.

Following the release of the 1987 video, the antigravity lean became so popular with audiences that Michael Jackson and his team undertook a search for ways to reproduce the move in live shows.

Because the use of wires is extremely difficult in front of a live audience, Jackson could not perform the move during his "Bad World Tour" in 1988, soon after the release of the music video (see "Michael Jackson - Smooth Criminal - Live Wembley 1988 - HD." Available at https://www.youtube.com/watch?v=dTXcBo86pz4). Undeterred, together with co-inventors Michael Bush and Dennis Tompkins, he developed an unusual shoe mechanism that, when used by dancers with strong core-strength training, could accomplish the antigravity lean without the use of wires. ${ }^{2}$ It took years to develop this invention, and the new technique was finally used in the 1992 "Dangerous World Tour." The success of the antigravity lean spurred on the use of this move in most of Jackson's later live shows featuring the song (see "Michael Jackson Lean Collection 1988-2009." Available at https://www.youtube.com/ watch? $v=b 2 J b Z P v 4 \operatorname{lgY}$ ). Inspired by Michael Jackson, the same shoe mechanism has been used by dancers all over the world who, after considerable training, have accomplished this move in front of mesmerized audiences without the use of wires (for example, see "Best Anti Gravity Lean compilation (the origins) Michael Jackson impersonator (Luca) 7 years old." Available at https://www.you tube.com/watch? $\mathrm{v}=\mathrm{zXGmdgSq0h8)}$.

It is this technique that we describe in our paper.

We are humbled by the attention that Mr. Kevin Pike has given our article through a Letter to the Editor ${ }^{3}$ and his provision of this interesting account of pop-music history. If what Mr. Pike writes is correct, it is possible that the idea of the shoe was inspired by MJ's inventiveness and modified to eliminate the piano wires for live shows. As a corollary, the authors wonder why Mr. Pike was not included as a co-inventor in the patent for the shoe mechanism that we have described. And if this is the "real history," as he has written in his letter, then perhaps a great injustice was done to $\mathrm{Mr}$. Pike and his team when the patent was filed.

\section{Acknowledgments}

We are grateful to Mr. Bunny Singh, medical illustrator, Postgraduate Institute of Medical Education and Research, Chandigarh, for his artistic illustrations. We sincerely thank Mrs. Shweta Yagnick, a dance critic and professional choreographer, for her valuable input in the article.

\section{References}

1. d'Hemecourt PA, Luke A: Sport-specific biomechanics of spinal injuries in aesthetic athletes (dancers, gymnasts, and figure skaters). Clin Sports Med 31:397-408, 2012

2. Jackson MJ, Bush ML, Tompkins D, inventors: Method and means for creating anti-gravity illusion. US patent 5,255,452. October 26, 1993.

3. Pike K: Michael Jackson and the "Smooth Criminal" music video: special effects. J Neurosurg Spine [epub ahead of print December 21, 2018. DOI: 10.3171/2018.9.SPINE181165] (Letter)

\section{Disclosures}

The authors report no conflict of interest. 